\title{
Surface Tension-like Forces Determine Bacterial Shapes: Streptococcus faecium
}

\author{
By A. L. $\mathrm{KOCH}_{1}{ }^{*} \mathrm{M}$. L. HIGGINS ${ }^{2}$ AND R. J. DOYLE ${ }^{3}$ \\ ${ }^{1}$ Program on Molecular, Cellular and Developmental Biology, Department of Biology, \\ Indiana University, Bloomington, Indiana 47405, U.S.A. \\ ${ }^{2}$ Department of Microbiology and Immunology, Temple University School of Medicine, \\ Philadelphia, Pennsylvania 19140, U.S.A. \\ ${ }^{3}$ Department of Microbiology and Immunology, University of Louisville, School of Medicine, \\ Louisville, Kentucky 40292, U.S.A.
}

(Received 29 April 1980; revised 25 September 1980)

The same tendency that causes soap bubbles to achieve a minimum surface area for the volume enclosed seems to account for many of the features of growth and division of bacteria, including both bacilli and cocci. It is only necessary to assume that growth takes place in zones and that only in these zones does the tension caused by hydrostatic pressure create the strain that forces the cell to increase the wall area. The stress developed by osmotic pressure creates strains that significantly lower the free energy of bond splitting by hydrolysis or transfer. We believe this is sufficient to make growing wall have some of the properties ordinarily associated with surface tension. The feature common to all bacterial cell wall growth is that peptidoglycan is inserted under strain-free conditions. Only after the covalent links have been formed are the intervening stressed peptide bonds cleaved so that the new unit supports the stress due to hydrostatic pressure.

The present paper analyses the growth of Streptococcus faecium in these terms. This is a particularly simple case and detailed data concerning morphology are available. The best fit to the data is achieved by assuming that growth takes place in a narrow region near the splitting septum and that the septal material is already under tension as it is externalized and is twice as thick as the external wall throughout the development of the nascent poles. Constancy of the ratio of hydrostatic pressure to the effective surface tension, $P / T$, is also consistent with electron microscopic observations.

\section{INTRODUCTION}

It is possible to blow soap bubbles of similar shape to the central growth zone of a typical Gram-positive coccus, Streptococcus faecium (Figs 1 and 2). This leads us to propose that in the growth zone the enlarging peptidoglycan wall has its shape determined by forces similar to surface tension forces. A model is presented here for this single case, but extensions of the model will be presented elsewhere that account for the ends of rod-shaped organisms, for the constant diameter of the cylindrical part of rod-shaped organisms, and for the growth process of the more nearly spherical staphylococci and gonococci. For $S$. faecium, the model assumes that the formation of the external peptidoglycan layer of the wall takes place mainly in a narrow zone near the developing septum. Previous work (Higgins \& Shockman, 1976) has shown that new wall growth occurs when the wall band splits and septal and pole material form between. As growth proceeds the septal ring narrows internally and splits externally and eventually leads to cell division. During an increment of time the increment of external 


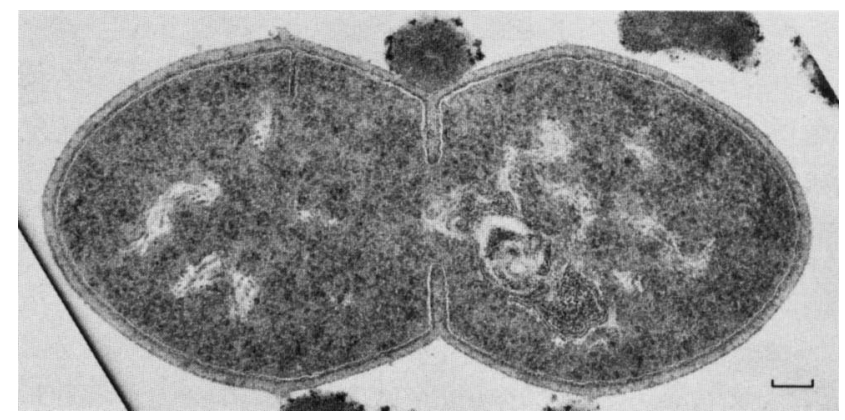

Fig. 1. Thin-section electron micrograph of a cross-section of Streptococcus faecium with a single growth zone. The bar marker represents $0 \cdot 1 \mu \mathrm{m}$.

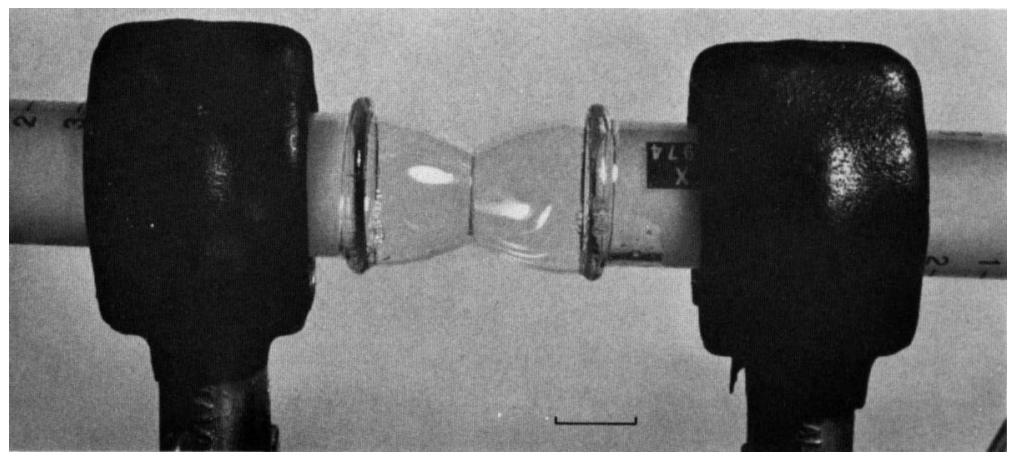

Fig. 2. Photograph of apparatus simulating the growth of Streptococcus faecium. The apparatus consisted of the barrels of two $5 \mathrm{ml}$ syringes connected in $\mathrm{T}$ to a constant, but adjustable source of pressure. The soap bubbles were blown, fused, and then the syringes were mechanically moved apart. As this was done the volume of the two soap bubbles increased simulating pole growth. Finally, the two bubbles separated. The photograph shows an intermediate stage. The bar marker represents $1 \mathrm{~cm}$.

peptidoglycan comes from two sources: newly formed glycan which is attached to the external wall mainly in the near vicinity of the splitting septum and the externalization of wall material previously present as septal material. The important assumption is made that this latter material was under tension before it was externalized and the work to develop that tension comes from metabolic processes occurring at an earlier stage. In any case, the septal material forms a ring that appears to be planar as if its inner circumference were under tension.

During an instant of growth, macromolecular synthesis and active transport increase the cell by a volume $\mathrm{d} v$. If the internal hydrostatic pressure is $P$, then an amount of work, $P \mathrm{~d} v$, is produced. This causes an increase in the external surface of the cell mediated by the cellular metabolic systems that carry out the formation of cell wall. By analogy to the physics of soap bubble formation (see Boys, 1890; Thompson, 1942), we define a surface tension, $T$, as the energy required to create a unit area of surface. The enzymic basis of this will be discussed below. The work done when increasing the surface by $\mathrm{d} A$ is $T \mathrm{~d} A$. For the soap bubble analogy this must equal the pressure-volume work, $P \mathrm{~d} v$ (Young, 1805; Laplace, 1806). As growth ensues the additional volume must be accommodated within the new surface. In Fig. 3 , a newly split wall band is depicted. The hatched area indicates the conical surface that accounts for half of the new area and contains half of the new volume. The slope of the conical regions determines the surface to volume ratio. 


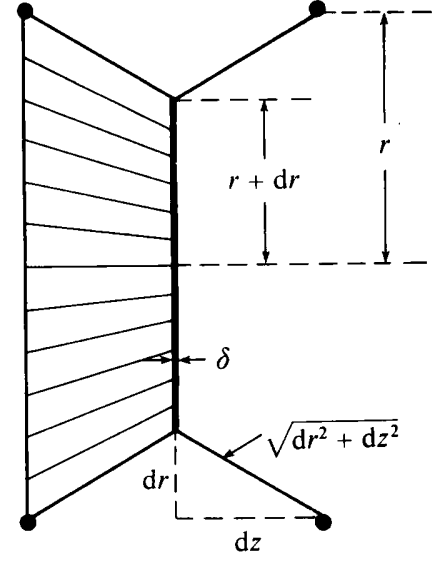

Fig. 3

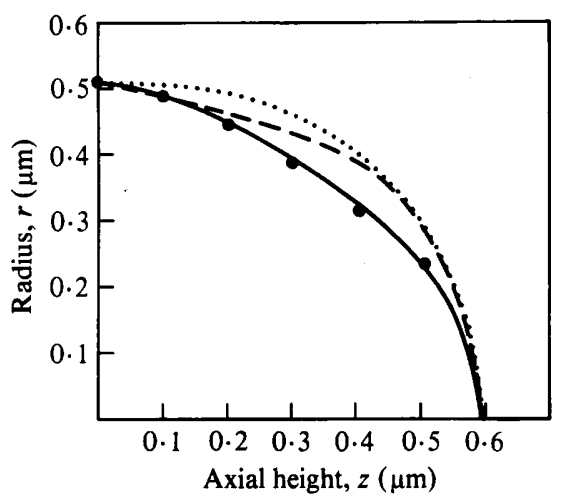

Fig. 4

Fig. 3. Geometric relationships in the region where new cell wall is forming. The figure depicts a wall band which had split an instant earlier. The hatched area on the left indicates one-half of newly formed external surface; it contains one-half of the volume increment, $\mathrm{d} v$. The thickness of the external wall is defined as unity and the septal wall as $\delta$ times thicker. The half-width of the growth zone is $\mathrm{d} z$. Because growth in this case leads to constriction the increment of radius, $\mathrm{d} r$, is negative as is the slope, $S=\mathrm{d} r / \mathrm{d} z$.

The combined new external area created by wall grawth on both sides of the septum is:

$$
2.2 \pi[r+(r+\mathrm{d} r)] \sqrt{\mathrm{d} r^{2}+\mathrm{d} z^{2}} / 2=4 \pi r \sqrt{1+S^{2}} \mathrm{~d} z
$$

The amount of external wall area produced from previous septal wall is:

$$
\delta \pi\left[r^{2}-(r+\mathrm{d} r)^{2}\right]=-\delta 2 \pi r \mathrm{~d} r=-\delta 2 \pi r S \mathrm{~d} z
$$

The difference between the two is the net area $\mathrm{d} A$. This expression is given in the text. The volume increment, $\mathrm{d} v$, enclosed by the newly created wall is:

$$
\mathrm{d} v=2 \pi|r+(r+\mathrm{d} r)|^{2} \mathrm{~d} z / 4=2 \pi r^{2} \mathrm{~d} z
$$

also given in the text. For the present case, it is assumed that immediately after growth has taken place the wall becomes rigid. Thus, the same diagram applies in the next instant of time, but $r$ is decreased from its previous value, and from its original value of $a$ at the time the wall band originally split.

Fig. 4. Shape of nascent poles of Streptococcus faecium. The points are experimental measurements taken from Higgins \& Shockman (1976). They are the average of measurement of 16 pairs of septated poles that were almost ready to separate. From measurements on many cells cultured and examined in the same manner, the mean value of $z_{\max }$ was $0.59 \mu \mathrm{m}$ and $a$ was $0.51 \mu \mathrm{m}$; these values inserted into equation (5) gave $P / T$ as 3.01 . Then these values were inserted into equation (4) to calculate the continuous line shown. This line was also computed with a mini-computer program described in the text that numerically integrates equation (3). The dashed line is the fit predicted by the assumption that the experimental points shown in Fig. 7 are valid measurements of $\delta$. Also shown is the i-driven growth curve (dotted line). In this case $\delta$ was originally set at 0.82 , and then the computer automatically raised $\delta$ the least amount that yielded a value permitting growth. The values of $\delta$ fitted in this way are shown by the dotted line in Fig. 7. The best fitted value of $P / T$ was $3 \cdot 85$.

Because septal material forms internally, the net change in surface area, $\mathrm{d} A$, depends on how much septal wall previously formed becomes externalized. The net surface area increment is the difference between the total area increment of external wall and the increment of area formed from the septal wall that has become externalized. From Fig. 3, it can be shown that $\mathrm{d} A=4 \pi r \sqrt{1+S^{2}} \mathrm{~d} z+\delta 2 \pi r S \mathrm{~d} z$. The dimensionless factor, $\delta$, is the thickness of the internal wall relative to the external wall. We have defined $S(=\mathrm{d} r / \mathrm{d} z)$ so that it is negative if the new growth causes constriction. The increment in cellular volume is $\mathrm{d} v=2 \pi r^{2} \mathrm{~d} z$. Substituting these expressions into the energy conservation equation: $P \mathrm{~d} v=T \mathrm{~d} A$, cancelling a factor of $2 \pi r \mathrm{~d} z$, and dividing by $T$, we obtain:

$$
\operatorname{Pr} / T=2 \sqrt{1+S^{2}}+\delta S
$$


This equation can be rearranged and then both sides squared to yield:

$$
\left(4-\delta^{2}\right) S^{2}+2 \delta(\operatorname{Pr} / T) S+4-(P r / T)^{2}=0
$$

Further simplification for certain values of $\delta$ is possible. For the particular case of the growth of streptococci, the thickness of the septal wall has been measured (Higgins \& Shockman, 1976) and found to reach twice the thickness of the peripheral wall at about the time that cell division occurs. For the case in which $\delta=2$, equation (2) becomes:

$$
S=(P r / 4 T)-(T / P r)=+\mathrm{d} r / \mathrm{d} z
$$

On the additional assumption that $P / T$ also remains constant throughout the time that a growth zone functions, this equation for the slope can be integrated to yield a relationship describing the shape of the growing pole:

$$
z=z_{\max }+(2 T / P) \ln \left[1-(\operatorname{Pr} / 2 T)^{2}\right]
$$

In this equation $z_{\max }$ is the height of the completed pole and is the value of $z$ where $r=0$. $z_{\max }$ is given by:

$$
z_{\max }=-(2 T / P) \ln \left[1-(P a / 2 T)^{2}\right]
$$

where $a$ is the radius of the base of the cone-shaped pole and is also the radius of the wall band at the initiation of the growth zone.

The fit of equation (4) to the electron microscopic observations of Higgins \& Shockman (1976) is shown in Fig. 4 by the continuous line: the value of $P / T(=3.01)$ was calculated by inserting the average values of $z_{\max }(=0.59 \mu \mathrm{m})$ and of $a(=0.51 \mu \mathrm{m})$ into equation (5), and then these values were inserted into equation (4). Since in this case there is little turnover of wall once it has been formed (Boothby et al., 1973) the good agreement supports the assumption concerning the conversion of pressure-volume work to the work in extending the net surface and the approximation that $\delta=2$ throughout the life of a growth zone.

\section{COMPUTER SIMULATION OF ZONE GROWTH}

A Wang 700 mini-computer was programmed to solve equation (2) for $S$, given $\delta, P / T$ and $r$. This basic program was modified for a number of purposes indicated below and in work to be reported elsewhere. In one mode the calculated value of $S$ was used to calculate the change in $r$, corresponding to a small increase in $z$. By making this calculation repeatedly with small steps of $z$, the computer calculated the shape of a growing pole given $\delta, a$ and $P / T$. The fit to equation (4) shown in Fig. 4 was checked in this way. The computer also calculated the contour length, pole area and pole volume. In another mode, the program tabulated $S$ for various ranges of values of $\delta$ and $\operatorname{Pr} / T$.

The slope of the nascent wall as a function of $\operatorname{Pr} / T$, calculated from equation (2), is shown in Fig. 5; various lines for chosen values of $\delta$ are presented. Notice that for values of $\delta$ less than 2, two slopes satisfy the equations for large enough values of $P r / T$, while the value of the slope is imaginary below critical values of $\mathrm{Pr} / T$. The critical value of $P r / T$ varies from 2 , for $\delta=0$, and approaches 0 as $\delta$ approaches 2. From inspection of Fig. 5, $\delta$ must become 2 when division takes place, since only then can $S$ become infinite, corresponding to the growth zone angle of $90^{\circ}$. A lesser angle would lead to cells with pointed ends. This requirement for $\delta$ to become 2 can be shown from equation (1) by setting $r=0$. After rearrangement and squaring, further rearrangement yields:

$$
S^{2}=4 /\left(\delta^{2}-4\right)
$$

Only when $\delta=2$ will the denominator vanish and $S$ become infinite.

The measurements of Higgins \& Shockman (1976) were made under special conditions (Toennies et al., 1961) in which chain formation was suppressed and the cells divided and separated from each other. Our model can explain the more usual chain-forming behaviour of 


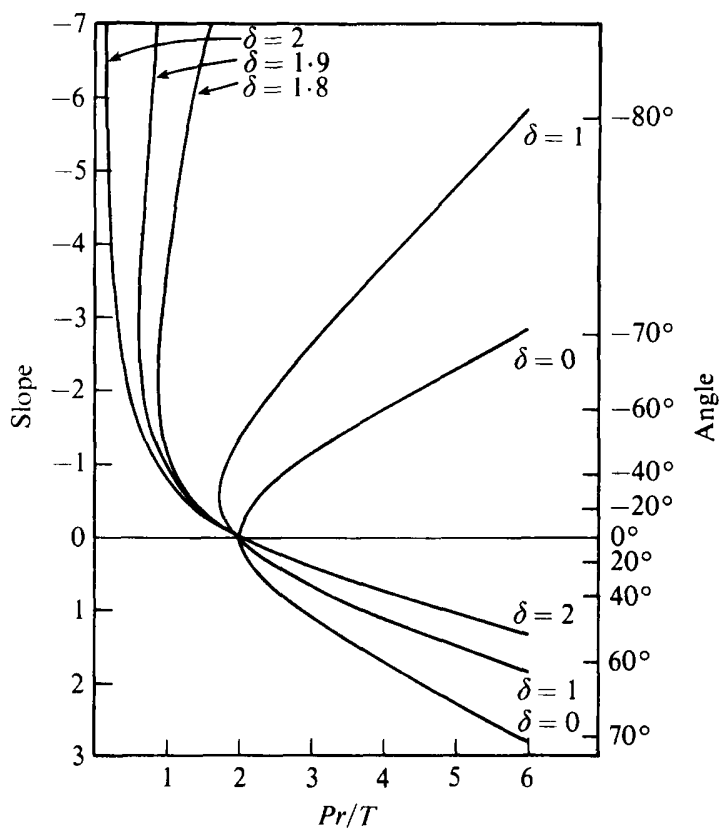

Fig. 5. The shape of newly formed wall. Computations based on equation (2) are shown. The slope and angle are taken as negative when they lead to constriction. Only for $\delta=2$ does the curve monotonically approach a slope of infinity (corresponding to a $90^{\circ}$ angle) as $\operatorname{Pr} / T$ approaches 0 . This condition is needed for the completion of cell division without pointed ends.

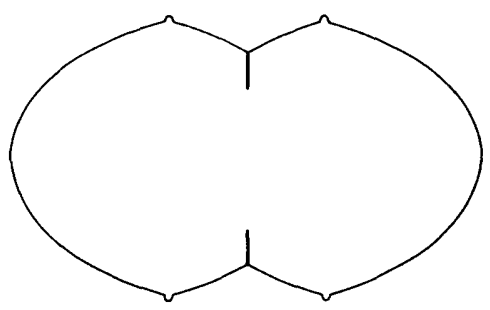

(a) $\delta=2$

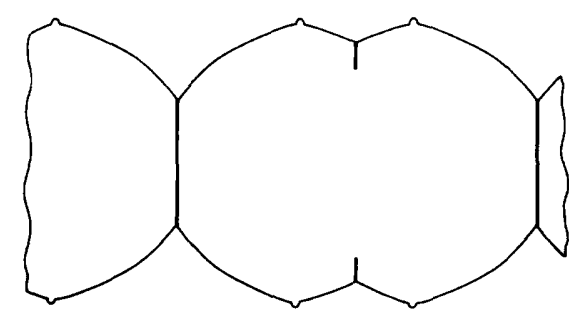

(b) $\delta=1.9$

Fig. 6. Theoretical shapes of streptococcal-type organisms. Calculations are shown for cells capable and incapable of completing cell separation. In $(a)$, where $\delta=2$, the model leads to repetitive cell division. The shape shown corresponds to a cell with a primary growth zone which will continue enlarging in volume and decreasing in septal radius, leading to two cells that may or may not have started secondary growth zones by the time of cell separation depending on the nutrient environment. If $\delta$ is less than 2 , as typified by $(b)$ where $\delta=1.9$, at some point in the development of the primary zone shown on the left, the numerical value of the solution to equation (2) became imaginary. Further new growth of cytoplasmic material will force the formation of secondary zones such as shown on the right. When this zone has grown to the shape of that shown on the left, further new growth of cytoplasmic material will force the formation of still other secondary zones such as shown at the right. When each zone has grown to the shape of that shown on the left, further growth is no longer possible. Eventually, the septa will finish closing yielding a chain of cocci.

many streptococci and staphylococci by postulating that in these cases, at least near the end of the life of a growth zone, $\delta$ is a little smaller than 2 . In such cases new growth must lead to constriction, but growth constriction could proceed only to a point. When the radius becomes sufficiently small so that $\operatorname{Pr} / T$ becomes critical, such that the value of $S$ would become imaginary, further growth of wall cannot proceed at that site. If $\delta$ cannot increase, this may 


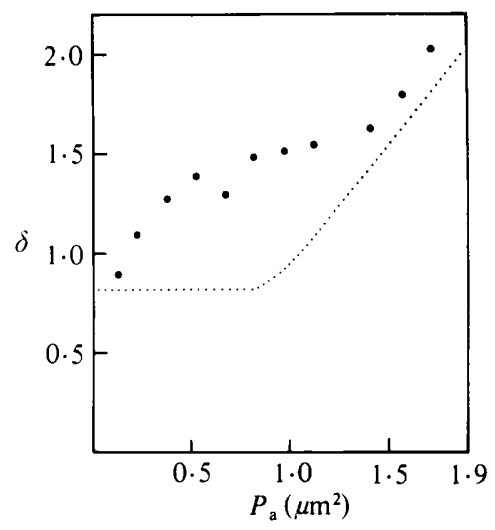

Fig. 7. Experimental and theoretical values of $\delta$. The points are the ratio $S_{\text {th }} / P_{\text {oth }}$ as measured and defined by Higgins $\&$ Shockman (1976). They are plotted as a function of the nascent pole area, $P_{\mathrm{a}}$. See the text for a discussion of the theoretical dotted line.

trigger the initiation of a new growth zone from the existing wall band(s) due to a momentary rise of hydrostatic pressure. Figure 6 shows the computer-simulated shape of chains of streptococci incapable of complete separation, for a case where $P$ and $T$ remain constant, and $\delta$ is constant at 1.9 throughout the life of a growth zone. Under these conditions, of course, the septum may close, separating the cytoplasm into two cells even if the septum does not split completely.

\section{i- DRIVEN GROWTH}

Although the assumption that $\delta$ is exactly 2 throughout growth provides a good fit to the shape of the nascent pole under the favourable conditions that lead to regular cell division, it is apparently not consistent with data. In Fig. 7 the observed ratio of the mid-distance thickness of the septum to the thickness of the split wall closest to the septum is plotted as a function of the nascent pole surface area. These data are taken from Higgins \& Shockman (1976) and belong to the same experimental series as the data of Fig. 4. It can be seen that in early phases, where $r$ is large, and the pole area small, this ratio is about 1 and increases progressively to 2 , just before cell division takes place.

If we assume this ratio to be an estimate of $\delta$, then $\delta$ is not at all constant. For this case the computer program was modified so that $\delta$ could be changed as the simulation proceeded. With the modified program the value of $\delta$ was changed systematically to agree with the observed ratio. (This could be readily done because the computer calculated nascent pole area as in the abscissa of Fig. 7.) Various $P / T$ values were assumed, and $P / T=3.66$ gave the best fit to the observed value of $z_{\max }(=0.59 \mu \mathrm{m})$. This simulation is shown in Fig. 4 by a dashed line. It can be seen that the fit is not as good as the fit made to the assumption that $\delta=2$ throughout (continuous line). We therefore suggest that the observed thickness of the septal wall at mid-distance is not relevant to the growth of external wall, but that in actuality there is a thickening of wall to $\delta=2$ in the immediate region of splitting.

The latter hypothesis is supported by the measurements of Higgins \& Shockman (1976) which indicated that the thickness of the peripheral wall nearest the septum was remarkably constant throughout a cycle of growth. Unfortunately there are no accurate measurements of the thickness of the septum just before the point where splitting is taking place.

The concept of a progressive increase in $\delta$ suggests another possible mechanism controlling wall growth; namely, that zonal growth causes invagination up to the point where the solution to equation (2) becomes imaginary. Then, growth can only proceed at that growth zone if the 
septal material becomes thicker so that $\delta$ increases enough for equation (2) to yield a unique real root. We call such a growth mechanism, i-driven growth because growth of external surface is driven by the thickening caused by $S$ becoming imaginary. Although, as we show below, this case does not seem to apply to $S$. faecium, it was a priori reasonable, and was developed for this case. It will be applied in work reported later to cocci that are more nearly spherical. Two approaches to i-driven growth were developed.

First, the computer program used above was modified in such a way as to simulate i-driven growth. Initially, a value of $\delta$ designated as $\delta_{0}$, together with the parameters $P / T$ and $a$, were inserted into the memory banks and then the computer solved equation (2); when the solution was real it computed a new value for $r$ from the slope. This cycle of calculations continued until the value of $\operatorname{Pr} / T$ decreased enough so that $\delta$ was imaginary; then the program added a small amount $(0.001)$ to $\delta$ and solved equation (2) again. Increasing $\delta$ only when necessary, the program described the development of the nascent pole with only initial assumptions of the value of $\delta_{0}$ and that growth only proceeds via the least possible thickening of the septum at the point of splitting.

The second approach is analytical and applies to the case when $\delta_{0}=0$. Both $\delta$ and $S$ can be calculated from $\mathrm{Pr} / \mathrm{T}$ for the case of i-driven growth. The condition that the two solutions of the quadratic of equation (2) are equal and real is that the square root in the quadratic formula be equal to 0 . From this relationship it follows that:

$$
\delta=\sqrt{4-(\operatorname{Pr} / T)^{2}}
$$

This value of $\delta$ can be substituted back into the quadratic formula to yield:

$$
S=\mathrm{d} r / \mathrm{d} z=-\sqrt{(2 T / P r)^{2}-1}
$$

This equation can be integrated on the assumption that $P / T$ is constant throughout the life of the growth zone. These manipulations yield:

$$
z=\sqrt{(2 T / P)^{2}-r^{2}}-\sqrt{(2 T / P)^{2}-a^{2}}
$$

If at the initiation of the growth zone, $\delta=0$, then $\delta$ will progressively rise to 2 as the nascent pole develops. Substituting $\delta=0$ when $r=a$ into equation (7) yields $2 T / P=a$, and then equation (9) becomes:

$$
z^{2}+r^{2}=a^{2}
$$

This is the equation of a circle, thus the pole will form in a spherical shape. If at the start of the growth process $\delta$ is greater than 0 , the growth will start with $\delta$ remaining at a constant value. Then, after some degree of constriction, i-driven growth commences and the remainder of the pole would be spheroidal. Figure 7 shows such a case (dotted line). $\delta_{0}$ was chosen as 0.82 and $P / T$ was adjusted to 3.85 to give the observed value of $z_{\max }$. It can be seen that this treatment fits both the observed values of $\delta$ and the pole shape poorly (see the dotted line in Fig. 4). Simulations with other values of $\delta_{0}$ gave even poorer fits.

On the whole, the data are best described by rejecting the i-driven growth model as well as the experimentally observed values of the thickness at the mid-distance of the septum. The assumption that $P / T=3.01$ and $\delta=2$ throughout the life of the growth zone accounts fully for the shape of the pole. This means that we must assume that the wall thickens with cell wall material as the septal wall splits into two and that the available electron microscopic measurements indicate the thickness of septal wall at some earlier stage than the actual septal splitting.

\section{GROWTH OF A SYSTEM UNDER INTERNAL PRESSURE}

The problem of enlarging a closed vessel under pressure must be foremost in any model for growth of the prokaryote wall. An essential part of our general model is that disaccharides or oligomers of peptidoglycan are covalently linked to stress-bearing wall with at least two 
covalent bonds before being subject to stress by hydrolysis of some stress-bearing bond. While it is self-evident that no stress is developed until at least two covalent bonds are made to existing wall, it is less obvious, but equally true, that to form a linkage that will be under tension at the instant it is completed will require extra work on the part of the biosynthetic machinery. To estimate the energies involved, let us calculate the stress that the bonds in the wall of an idealized coccus of perfectly spherical shape with a single monolayer of peptidoglycan would experience if all possible covalent bonds were formed and all shared the stress equally.

While data are very few, the Gram-negative rod Escherichia coli has about 4 to 5 atm of osmotic pressure. This range is only approximate; it includes the values measured 25 years ago by Mitchell \& Moyle (1956) and recent measurements by Stock et al. (1977). We will assume a value of $5 \mathrm{~atm}$ for our idealized organism. The osmotic pressure causes an equal hydrostatic pressure as water tends to enter the cell. This pressure pushes the cytoplasmic membrane against the peptidoglycan covering of the cell and creates a tension in the layer tending to expand this layer of the wall. The tension so developed would be almost totally in the glycan layer, because of the fluid nature of the cytoplasmic layer (or an outer membrane layer, if present).

Gram-positive organisms have a much greater osmotic pressure (approximately $20 \mathrm{~atm}$ ) and also a much thicker layer (approximately $20 \mathrm{~nm}$ ) of peptidoglycan, which may consist of about five layers each $4.5 \mathrm{~nm}$ thick. On this basis, osmotic forces have approximately equally important consequences at the level of the individual bonds forming the wall in Gram-positive as Gram-negative organisms.

The energy conservation formula, $P \mathrm{~d} v=T \mathrm{~d} A$, when applied to a sphere of radius $r$, becomes $P=T / 2 r$. The osmotic pressure of $5 \mathrm{~atm}$ creates in a spherical shell with an assumed radius of $0.7 \mu \mathrm{m}$ a tension of $300 \mathrm{dyn} \mathrm{cm}^{-1}$. From the dimensions of the peptidoglycan structure obtained by X-ray diffraction, the chains of carbohydrate are packed parallel to each other with a spacing of $0.45 \mathrm{~nm}$ in microcrystalline regions (Labischinski et al., 1979). Therefore, in the plane of the cell wall and perpendicular to the axis of the chains there would be $2.2 \times 10^{7}$ chains $\mathrm{cm}^{-1}$. Scission of a single carbohydrate chain would therefore relieve locally a stress of $300 /\left(2.2 \times 10^{7}\right)=1.35 \times 10^{-5}$ dyn and relieve strain in all parts of the uncross-linked region of the chain in the vicinity of the cut. This strain would then be distributed among other chains.

In both the chemical and biochemical reactions which could lead to hydrolysis the covalently bound atoms of the bond that is to be cleaved must move further apart by about $0.05 \mathrm{~nm}$, in the formation of a 'reactive' activated intermediate complex. From Fig. 8, it is clear that the lowering of the energy of activation due to strain depends not on the shape of the potential energy curve but only on the amount of stretching involved in the formation of the activated complex; i.e., simply on force times distance. The stress of $1.35 \times 10^{-5}$ dyn calculated above operating over a distance $0.05 \mathrm{~nm}$ corresponds to an energy of $0.97 \mathrm{kcal} \mathrm{mol}^{-1}$ or $4.06 \mathrm{~kJ} \mathrm{~mol}^{-1}$. Typical values of the free energy of hydrolysis of glycosidic bonds are in the range of -12 to $-18 \mathrm{~kJ} \mathrm{~mol}^{-1}$ and thus the stability of a glycoside bond under the tension that would normally be present in a cell wall is significantly decreased and the free energy of hydrolysis would be increased by $-4.06 \mathrm{~kJ} \mathrm{~mol}^{-1}$. Of course, in aqueous solution glycosidic bonds would hydrolyse eventually because the free energy of formation is negative, but the more relevant question concerns the rate and not the extent of hydrolysis. In order to hydrolyse such bonds, energy must temporarily be associated with the bond to create a less stable intermediate 'activated' state in which the original bond is strained. Energies of activation are reduced by enzymes, and that is the mode by which they direct cellular metabolism. Evidently, external mechanical stress would also lower the energy of activation. Figure 8 shows that if the tension does not alter the bond geometry, the energy of activation is lowered as much as the free energy of hydrolysis. This would cause the rate of the hydrolysis reaction to increase 4.83 -fold at $37^{\circ} \mathrm{C}$ under the stress present in our hypothetical cell. 


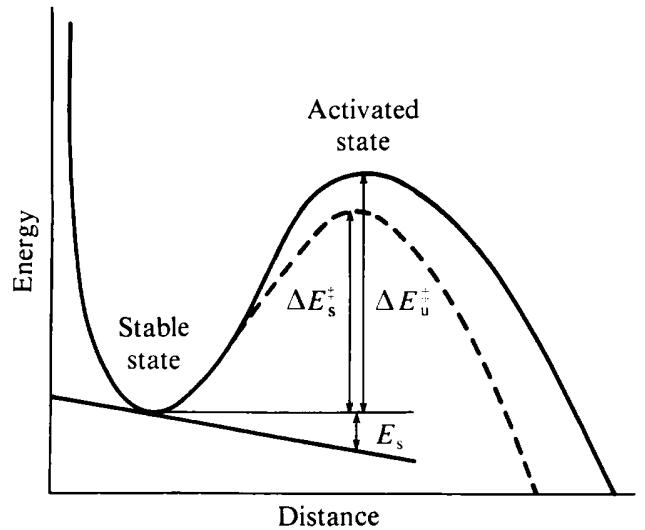

Fig. 8. Effect of stress on the energy of activation. This graph shows a hypothetical potential energy curve for a chemical reaction. The abscissa is the distance between two cleavable atoms in the macromolecule. In order to break or make this bond the atoms must pass through an energy maximum. When the bond is stressed, work is done on the bond to increase its length. Once the bond is stretched past its activated state it is broken and no more work is done as the atoms are moved yet further apart. This work supplied by the stress simply depends on force and the distance between the stable position and activated state and not on the detailed quantum mechanical properties of the bond; i.e. $\Delta E_{\mathrm{s}}^{\dagger}=\Delta E_{\mathrm{u}}^{\dagger}-E_{\mathrm{s}}$, where $\Delta E_{\mathrm{s}}^{\dagger}$ and $\Delta E_{\mathrm{u}}^{\dagger}$ are the energies of activation for the unstressed and stressed bond and $E_{\mathrm{s}}$ is the strain energy equal to the product of force times the distance the atoms are moved apart.

In the walls of bacteria, the glycosidic bonds do not continue all around the cell but average only 5 to 50 disaccharide units in length, i.e. less than $2 \%$ of the circumferential distance. For nearly spherical bacteria the chain length is 5 to 16 (Oldmixon et al., 1974). Because of autolytic enzyme action, these lengths are low but probably they are underestimates by no more than factors of two- to fourfold. Therefore, because of these interruptions in the chains, the stress on the remaining bonds is greater than calculated and they should be still more readily hydrolysed. The exact stress would depend on the detailed geometry of the stress-bearing bonds surrounding the bond under consideration.

It is not so easy to calculate the stress on bonds perpendicular to the glycan chains in the plane of the cell surface. The chains are held together with many weak hydrogen bonds between the carbohydrates and by the less numerous covalent links between the peptide tails of not necessarily adjacent glycan chains. The hydrogen bonds should be easily broken because they are weaker and the wall is essentially porous and immersed in an aqueous environment. This must allow the facile breaking of glycan-glycan hydrogen bonds and formation of hydrogen bonds between the polysaccharide and water. Then, hydrogen bonds between glycan residues may form anew with the same or another segment of the wall and thus respond to the stretch permitted by the covalent linkages. Consequently, hydrogen bonds probably share very little of the strain. This means that nearly the full stress is on the peptidyl links in the direction perpendicular to the glycan chains. At most there could be one bridge every $2.06 \mathrm{~nm}$ along the carbohydrate chain if alternate peptide side-chains went in exactly opposite directions and were tautly connected to other chains. While alternate positioning of residues is characteristic of cellulose and chitin, the structural materials of plants and insects, in bacteria the directions appear to be random (Labischinski et al., 1979). Some protrude upwards, some downwards and some protrude in the plane of the wall. What is more relevant is that only a fraction of the chains are engaged in cross-linkage. Consequently, the number of bonds sharing the tension in a plane perpendicular to the carbohydrate chain is approximately 10 -fold lower and the effect of stress in lowering the free energy of hydrolysis is about 10-fold higher than calculated above. Lowering the energy of activation by $45 \mathrm{~kJ} \mathrm{~mol}^{-1}$ would, of course, have profound influence on rates of reaction of $\mathrm{N}$-acetylmuramoyl-L-alanine amidases and other enzymes that can cleave the peptide chains. 
To the extent that the wall contains other substances. such as teichoic acid, that have only single covalent links to the wall, the number of stress-bearing bonds in a region is decreased and the stress on each remaining strained bond is increased.

\section{POTENTIAL MODES OF CELL WALL ENLARGEMENT}

We suggest that the only way new peptidoglycan can be introduced into the wall of growing organisms is in the stress-free state and only later do the bonds become structurally important and bear the stresses due to osmotic pressure. Logically, there are three ways that strain-free insertion can occur and all three may occur in prokaryotes. These methods are analogous to the ways employed by man to build dams, dig tunnels through mud, or repair a rip in the hull of a ship.

The first way (relevant to cell division of both bacilli and cocci of both Gram-positive and Gram-negative organisms) is simply to build a cross-wall with material in a stress-free (or stress-reduced) region inside the cell, i.e. in the region involved in the septation needed to convert one cell to two. Under the condition that the external surface on which the septation starts is rigid the newly inserted wall material will not be under stress. The septum forms as a planar ingrowth area. Therefore, the osmotic pressure cannot produce a strain on the newly formed bonds. The mathematical treatment tacitly assumed that the septum is rigid and not appreciably stretched when tension is placed on it. The septal peptidoglycan only comes under stress just before the septum splits to that point.

There are two ways that the septal wall could be formed with these properties. First, the septal material may be sufficiently cross-linked to become rigid. The second way is that the septal wall is formed in a different way from additions to the external wall and that glycan polymers form as complete rings or nearly complete rings, all around the closing septum. They might be oriented by hydrogen bonding to the more peripheral ring of glycan. Thus, glycan molecules would not be capable of much expansion when tension came to be applied by the splitting process. Obviously, such paracrystalline structure would be lost as the cell wall material was re-worked with new peptidoglycan in the act of becoming externalized. In any case, after the more peripheral material splits and is externalized. tension is developed in the then outer surface of intact septum (just internal to the splitting material), but no appreciable expansion occurs. Consequently $\mathrm{d} A$ will be 0 or at least very small and little or no work of the $T \mathrm{~d} A$ kind will be done.

The mode of enlargement (also appropriate for the extension of the cylindrical portion of wall in Gram-negative rods) is to form strain-free bonds linking new oligo-peptidoglycan to the existing rigid structure by transpeptidation with the unused $\mathrm{D}$-alanine peptide bonds. If the bridges are formed at random then loose networks will be formed surrounding an area under stress (Fig. 9). Only after the new oligopeptides are secure do the enzymes present in the wall carry out the cleavage of bonds of the pre-existing wall under stress. This requires sophisticated hydrolytic enzymes which cleave bonds under tension only if there is a network of unextended, unstressed peptidoglycan surrounding the bonds to be cleaved. Once they are cleaved the stress appears on the newly linked peptidoglycan oligomers and they become extended and pulled into the plane bearing the stress. This method of insertion would be adaptable to growth over the entire surface (diffuse growth) or to growth in particular growth zones. In this mode, hydrostatic pressure would create the tension lowering the energy of activation for the cleavage of old peptidoglycan that limits the enlargement of the cell wall.

The third mode of enlargement which can proceed without stress on newly inserted oligomers of peptidoglycan is inside-out growth. We mention it here only for completeness; it is applicable to extension of the cylindrical region of Gram-positive rods. In this mode of growth, peptidoglycan oligomeric units are inserted on the inner face of the wall just outside of the cytoplasmic membrane. This mode requires the continuous dissolution of the external surface of the wall. This leads to a loose network covering the cytoplasmic membrane but not bearing stress. This mode will be discussed in detail in a future communication. 


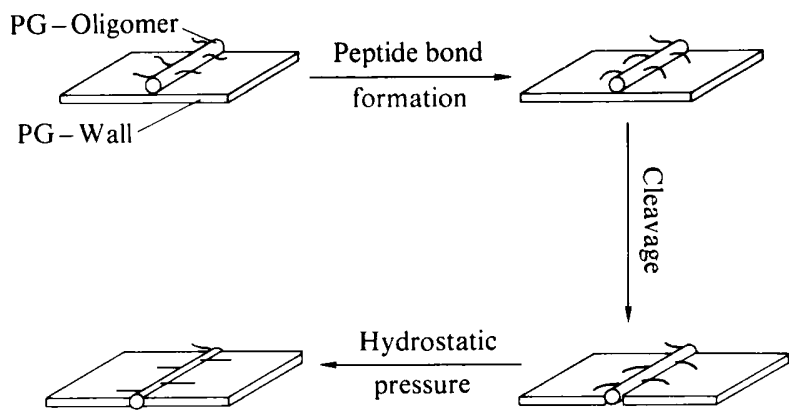

Fig. 9. Addition of new material to an external wall already under tension. Peptidoglycan (PG) oligomers are first covalently attached via their peptide cross-bridges to the existent, stressed wall. Such linkages, if formed at random, would leave the newly inserted unit loosely attached, i.e. not in an extended conformation. If the hydrolytic or transferase enzymes acted only on the bonds under stress and only in a region surrounded by the newly formed linkages, then the newly inserted unit would be pulled into the wall. It would bear the stress due to hydrostatic pressures and this would cause the wall to expand and the newly inserted peptidoglycan to have an extended conformation.

Finally, we would like to note that the specific model presented here can be made suitable for other cases by modifying the assumptions so that the pressure, tension and septal wall contribution may change as the cell cycle proceeds. The model can also be extended to growth zones that are not narrow or not circumferential. It is simply that the growth zone of $S$. faecium appears to be narrow, that it is located solely at the splitting region of the septum, that $P, T$ and $\delta$ are constant, and that $\delta=2$. These halcyon circumstances greatly simplify the interpretation of this particular type of bacterial growth.

Dr Lolita Daneo-Moore served a key role in bringing the authors to focus on this problem. We wish to thank Hiroshi Nikaido and David Mirelman for organizing the Gordon conference where many of the ideas presented here gelled. Work in our laboratories is supported by PHS SO7 RR 7031 and NSF PCM 7911241 (A.L.K.); NIH AI- 10971 (M.L.H.): and NSF PCM 78-08903 (R.J.D.).

\section{REFERENCES}

Boothby, D., Daneo-Moore, L., Higgins, M. L., Coyette, J. \& Shockman, G. D. (1973). Turnover of bacterial cell wall peptidoglycan. Journal of Biological Chemistry 248, 2161-2169.

Boys, C. V. (1890). Soap Bubbles and the Forces which Mould Them. Society for the Promotion of Christian Knowledge. Reprinted by Dover, New York (1959).

Higgins, M. L. \& Shockman, G. D. (1976). Study of a cycle of cell wall assembly in Streptococcus faecalis by three-dimensional reconstructions of thin section of cells. Journal of Bacteriology 127, 1346-1358.

Labischinski, H., BARNickel, G., Bradaczek, H. \& GiesBrecht, P. (1979). On the secondary and tertiary structure of murein. European Journal of Biochemistry 95, 147-155.

Laplace, P. S. (1806). Mécanique Céleste. Paris: Imprimeur Imperiale.

Mitchell, P. \& Moyle, J. (1956). Osmotic function and structure in bacteria. Symposia of the Society for General Microbiology 6, 150-180.

Oldmixon, E. H., Glauser, S. \& Higgins, M. L. (1974). Two proposed general configurations for bacterial cell wall peptidoglycans shown by spacefilling models. Biopolymers 13, 2037-2060.

Stock, J. G., Rausch, B. \& Roseman, S. (1977). Periplasmic space in Salmonella typhimurium and Escherichia coli. Journal of Biological Chemistry 252, 7850-7861.

Thompson, D'Arcy, W. (1942). On Growth and Form, 2nd edn, pp. 351-384. Cambridge: Cambridge University Press.

Toennies, G., ISZARD, L., Rogers, N. B. \& Shockman, G. D. (1961). Cell multiplication studied with an electronic particle counter. Journal of Bacteriology 82, 857-866.

Young, T. (1805). Cohesion of fluids. Philosophical Transactions of the Royal Society 95, 65-87. 